\section{THE PROGRAM OF PREVENTION AND CONTROL OF THE VERTICAL TRANSMISSION OF THE HIVIAIDS IN CUBA (JANUARY 1986-DECEMBER 2009)}

\author{
I. Gonzalez-Nuñez ${ }^{1}$, M. Diaz- Jidy ${ }^{2}$ \\ ${ }^{1}$ Atencion Medica, Instituto de Medicina Tropical \\ Pedro Kouri, La Habana, ${ }^{2}$ Atencion Medica, \\ Instituto de Medicina Tropical Pedro Kouri, Ciudad \\ de la Habana, Cuba
}

Background and aims: Since 1986, a controlled program has been established in the primary health care system in order to reduce HIV vertical transmission in Cuba.

Methods: The usual approach since 2008, applied to every HIV+ pregnant woman who decides to keep her pregnancy, is to administer HAART independently of her immunological status, from week 14 to the time when the caesarean section is carried out (week 38). Breastfeeding is strongly discouraged. The newborn child receives ZDV $(2 \mathrm{mg} / \mathrm{Kg} / \mathrm{dose})$ every 6 hours for the first 6 weeks. The children are followed-up at the IPK outpatient office, where their HIV infection status is determined. Infected children are treated with antiretrovirals according to the presence of opportunistic diseases, CD4 cell count and viral load.

Results: A total of 2321 seropositive women have been reported since January 1, 1986 to December of $2009(18,9 \%)$ of all the seropositive cases of the country (2321 / 12217), $356(15,3 \%)$ have given birth a total of 420 children (26 women have delivered twice and 6 have twins); 36 of 420 are HIV+ $(8,5 \%)$, 34 classified as AIDS (34/36=94,4\%) , 23 are under treatment with HAART ; 2 are asymptomatic and $11(11 / 36=30,5 \%)$ have died. No infection was demonstrated in 292 children by PCR and Western Blot $(292 / 420=69,5 \%)$ and $92(92 / 420=21,9 \%)$ are still under study.

Conclusion: The Program for Prevention and Control of HIV Vertical Transmission is effective since the number of infected children is low, similar to the figures reported for developed countries.

\section{BACTERIAL INFECTIONS IN CHILDREN UNDER 14 AND DETECTION OF ANTIBIOTICS RESISTANCE PATTERNS OF ISOLATES IN HAMADAN}

\author{
R. Yousefi Mashouf ${ }^{1}$, F. Eghbalian², M. Koshki \\ ${ }^{1}$ Microbiology, ${ }^{2}$ Pediatrics, ${ }^{3}$ Hamadan University of \\ Medical Sciences, Hamadan, Iran
}

Background and aims: Bacterial infections are one of the main problems for pediatric diseases in the third world countries. Therefore, the aim of study was the evaluation of frequency of bacterial infections in children and detection of antibiotics resistance patterns of bacteria in Hamadan, West of Iran.

Methods: This study was performed on 6391 children under 14 years of age who admitted at pediatric wards in Ekbatan hospital in Hamadan city. All children which were diagnosed with pneumonia, meningitis, septicemia, gastroenteritis, and urinary tract infections (UTI) were evaluated. Disk agar diffusion method was used to determine the isolated bacterial resistance to 12 antimicrobial agents. Data were analyzed using spss system.

Results: From 6391 samples, $27.7 \%$ were positive culture. $65.4 \%$ of isolated bacteria were gram negative and $34.6 \%$ (613) were gram positive bacteria. The most common infections were: urinary infections (36.8\%), gastroenteritis (34.9\%), sepsis $(17 \%)$, pneumonia $(9.2 \%)$, and meningitis $(2.1 \%)$. Isolated bacteria were: E. coli $(36.3 \%)$, Staphylococcus aureus (18.2\%), Staphylococcus epidermidis (13.3\%), Klebsiella spp.,(10\%), Enterobacter spp., (6\%), Shigella spp., (3.9\%), Pseudomonas auroginosa (2.8\%). The most effective antibiotics on both gram positive and gram negative isolates were ceftriaxone, nitrofurantoin, cefepime, kanamycin and gentamicin. Most strains were resistant against cephalexin, ampicillin, erythromycin and co-trimoxazole.

Conclusions: This study showed that the most common bacterial infections were gastroenteritis, UTI and sepsis. E.coli and Kelebsiella spp., were the most common gram negative bacteria and Staphylococcus aureus was the most common gram positive one, which were resistant to the wide spectrum antibiotics. 